\title{
THE RELATIVE COMPLETION OF AN $A$-SEGAL ALGEBRA IS CLOSED
}

\author{
JAMES T. BURNHAM
}

\begin{abstract}
The main result is this theorem: If the Banach algebra $A$ has bounded approximate right units and $B$ is an $A$-Segal algebra, then the relative completion of $B$ with respect to $A$ is an $A$-Segal algebra. Furthermore, $B$ is a closed ideal of its relative completion with respect to $A$.
\end{abstract}

In this paper we define the relative completion of an $A$-Segal algebra and present an analysis of the resulting object. At the heart of our analysis is this fundamental result: In the presence of approximate units an $A$-Segal algebra is a closed ideal in its relative completion and the relative completion is itself an $A$-Segal algebra. This and the other results presented in this paper were announced in [4]. Furthermore, we will use the results in this paper in our analysis (done jointly with Richard R. Goldberg) of the multiplier theory of Segal algebras on locally compact groups [5].

It is of interest to compare the results in this paper with work of N. Th. Varopoulos [16].

Conventions. Algebras have no identity, and ideal means left ideal unless something to the contrary is explicitly stated. The reader will easily see how to formulate and prove "right-hand" versions of our theorems.

We begin our work with a statement of the definition of $A$-Segal algebra.

Definition 1. Let $\left(A,\|\|_{A}\right)$ be a Banach algebra. We say the subalgebra $B \subseteq A$ is an $A$-Segal algebra provided (i) $B$ is a dense ideal of $A$ which is a Banach algebra with respect to the norm \|\|$_{B}$, and (ii) the natural injection of $B$ into $A$ is continuous and multiplication is (jointly) continuous from $A \times B$ into $B$.

Remarks 2. Part (ii) of 1 implies the existence of constants $K, M>0$ so that \|\|$_{A} \leq K\|\|_{B},\|f \cdot g\|_{B} \leq M\|f\|_{A}\|g\|_{B}$ for all $f \in A$ and all $g \in B$. Although these latter inequalities are our basic tools, the phrasing in Def-

Received by the editors December 12, 1973.

AMS (MOS) subject classifications (1970). Primary 46H25; Secondary 43 A15.

Key words and phrases. Banach algebras, Banach modules, closed ideals, approximate identities, Segal algebras. 
inition 1 makes sense for topological algebras that are not necessarily normed algebras. The basic theory for the more general situation is indicated in [3] and many of the results herein are valid in the more general setting. Appealing to a result of M. A. Rieffel [14] we may and do take the constant $M$, appearing in the product inequality, to be one. Finally, as the results of the present paper constitute contributions to the structural aspects of $A$-Segal algebras, we point out that a great deal is already known about the structure of $A$-Segal algebras: [1]-[4], [6]-[9], [11], [13], [15].

Definition 3. Let $B$ be an $A$-Segal algebra. The relative completion of $B$ with respect to $A$, denoted $\widetilde{B}^{A}$, is defined in this way:

$$
\widetilde{B}^{A}=\bigcup_{\eta>0} \overline{S_{B}(\eta)}{ }^{A},
$$

where $S_{B}(\eta)=\left\{f \in B \mid\|f\|_{B} \leq \eta\right\}$ and $\bar{E}^{A}$ is the closure of $E$ in the $A$-norm. A norm on $\widetilde{B}^{A}$, denoted $\|\mid\|$, is defined in this way:

$$
\|f\|=\inf \left\{t \mid f \in{\overline{S_{B}(t)}}^{A}\right\} .
$$

The following lemma provides us with a useful tool to be used in our analysis of $\widetilde{\sim}^{A}$.

Lemma 4. Let $B$ be an A-Segal algebra. If $f \in \widetilde{B}^{A}$, then there exists a sequence $\left\langle f_{n}\right\rangle$ in $B$ such that

(i) $\left\|f_{n}-f\right\|_{A} \rightarrow 0$, and

(ii) $\left\|f_{n}\right\|_{B} \rightarrow\|f \mid\|$.

Proof. If $f \in \widetilde{B}^{A}$ and $\alpha_{k} \equiv\|f\| \|+1 / k, k=1,2, \cdots$, then $f \in \overline{S_{B}\left(\alpha_{k}\right)}{ }^{A}$. Hence, there exists $f_{n} \in S_{B}\left(\alpha_{n}\right)$ so that $\left\|f_{n}-f\right\|_{A}<1 / n, n=1, \cdots$, and so (i) follows. Now $\left\|f_{n}\right\|_{B} \leq \alpha_{n}$ and so we see that

(iii) $\operatorname{Lim} \operatorname{Sup}_{n \rightarrow \infty}\left\|f_{n}\right\|_{S} \leq\|\| f \|$.

Suppose, for some $\delta$ that $\operatorname{Lim} \inf _{n \rightarrow \infty}\left\|f_{n}\right\|_{B}<\delta<\|f \mid\|$. Then some subsequence of $\left\langle f_{n}\right\rangle$ lies in $S_{B}(\delta)$. This and (i) would imply that $f \in{\overline{S_{B}(\delta)}}^{A}$, contradicting $\delta<\|f\| \|$. Hence, no such $\delta$ exists and so

$$
\underset{\eta \rightarrow \infty}{\operatorname{Lim} \inf }\left\|f_{n}\right\|_{B}=\|f\| \|
$$

This and (iii) show that (ii) holds.

Here is an example of an $A$-Segal algebra in its relative completion. We set $A=L^{1}(G)$ and $B=C(G)$ where $G$ is an infinite compact group. In this case $\widetilde{C(G)} L^{1}=L^{\infty}(G) \neq C(G)$. We shall give further examples after some basic results have been established. 
Now it is well known that the relative completion of a Banach space is again a Banach space [10]. In our situation more is true. Indeed, $\widetilde{B}^{A}$ is an $A$-Segal algebra. N. Th. Varopoulos [16] has shown that $B$ need not be closed in $\widetilde{B}^{A}$. However, if $B$ and $A$ share right approximate units which have $A$ norm one then $B$ is closed in $\widetilde{B}^{A}$ and the embedding is an isometry. Furthermore, in the presence of right approximate units $\widetilde{B}^{A}$ has a simple description which is of interest in its own right (compare Theorem 5 below with [12, 35.11, p. 380]).

Convention. For the remainder of this paper we suppose that $B$ and $A$ have common right approximate units, $E$, with $\|e\|_{A}=1$ for all $e \in E$.

Furthermore, we suppose that

$$
\|f e\|_{B} \leq\|f\|_{B}\|e\|_{A}=\|f\|_{B} \quad(f \in B, e \in E) .
$$

Note that this inequality is not a consequence of the definition of $A$-Segal algebra because $B$ need not be a right ideal! This condition is satisfied in all the examples we know of.

Theorem 5. If $B$ is an A-Segal algebra, then the following two conditions are equivalent:

(1) $f \in \widetilde{B}^{A}$;

(2) $M=\operatorname{Sup}\left\{\|f e\|_{B} \mid e \in E\right\}<\infty$.

If either condition bolds then $M=\|\| f \|$.

Proof. Suppose $f \in \widetilde{B}^{A}$. By Lemma 4 there exists a sequence $\left\langle f_{n}\right\rangle$ in $B$ so that $\left\|f_{n}-f\right\|_{A} \rightarrow 0$, and $\left\|f_{n}\right\|_{B} \rightarrow\|f\|$. Then, for each $e \in E$ we have $\left\|f_{n} e-f e\right\|_{B} \leq\left\|f_{n}-f\right\|_{A}\|e\|_{B} \rightarrow 0$ as $n \rightarrow \infty$. Thus, $\|f e\|_{B}=\operatorname{Lim}_{n \rightarrow \infty}\left\|f_{n} e\right\|_{B} \leq$ $\operatorname{Lim}_{n \rightarrow \infty}\left\|f_{n}\right\|_{B}=\|f\|$. Hence, (2) holds and $M \leq\|f\|$. Now suppose (2) holds for some $f \in A$. Choose an ( $f$-dependent) sequence $\left\langle e_{n}\right\rangle$ from $E$ so that $\left\|f e_{n}-f\right\|_{A} \rightarrow 0$. By hypothesis $f e_{n} \in S_{B}(M)$. Hence, $f \in \widetilde{B}^{A}$ and $\|f\| \leq M$. Hence (1) holds. But then $M \leq\|f\| \|$ so that $M=\|f\| \|$.

Theorem 6. If $B$ is an A-Segal algebra, then $\widetilde{B}^{A}$ is an A-Segal algebra. Furtbermore, $B$ is a closed ideal of $\widetilde{B}^{A}$ with $\|f\|_{B}=\|f\|$ for all $f \in B$.

Proof. We have already noted that $\widetilde{B}^{A}$ is an $A$-Segal algebra. As Theorem 5 permits a simplified proof we include the details.

Suppose $f, g \in \widetilde{B}^{A}, \alpha, \beta$ are complex numbers. Then

$$
\|(\alpha f+\beta g) e\|_{B} \leq|\alpha|\|f e\|_{B}+|\beta|\|g e\|_{B} \leq|\alpha|\|f|\|+|\beta|\| g\|\| .
$$

Taking Sup over $e \in E$ shows that $\widetilde{B}^{A}$ is a normed linear space. Suppose 
now that $\left\langle f_{n}\right\rangle$ is Cauchy in $\widetilde{B}^{A}$. Clearly \|\|$\geq\|\|_{A}$ so that $\left\langle f_{n}\right\rangle$ is Cauchy in $A$. Whence $\exists f \in A$ so that $\left\|f_{n}-f\right\|_{A} \rightarrow 0$. Given $\epsilon>0$ choose $N$ such that $\left\|f_{m}-f_{n}\right\|<\epsilon(m, n \geq N)$. Fix $e \in E$. Now $\left\|\left(f_{m}-f_{n}\right) e\right\|_{B} \leq \epsilon$ and $\left\|\left(f_{n}-f\right) e\right\|_{B} \leq\|e\|_{B}\left\|f_{n}-f\right\|_{A} \rightarrow 0$ so that letting $n \rightarrow \infty$ in the first inequality we obtain $\left\|\left(f_{m}-f\right) e\right\|_{B}<\epsilon(m \geq N)$. Thus, by Theorem 5 we get $f_{m}-f \in \widetilde{B}^{A}$. Hence $f \in \widetilde{B}^{A}$ and $\left\|f_{m}-f\right\|<\epsilon$ so that $\widetilde{B}^{A}$ is complete. Suppose now that $f \in A, g \in \widetilde{B}^{A}$. Then $\|f g e\|_{B} \leq\|f\|_{A}\|g e\|_{B}$. By Theorem 6 we get $\|f g e\|_{B} \leq\|f\|_{A}\|g\|$ and again by Theorem 5, fg $\in \widetilde{B}^{A}$. Taking Sup over $e \in E$ gives the module inequality and we have shown that $\widetilde{B}^{A}$ is an $A$-Segal algebra.

We will now show that $B$ is a closed ideal of $\widetilde{B}^{A}$ and $\|f\|_{B}=\|f\| \|$ for all $f \in B$. We first show that $\|f\|=\|f\|_{B}$ for all $f \in B$. If $f \in B$, then $f \in$ $\widetilde{B}^{A}$ so that $\|f e\|_{B} \leq\|f\|(e \in E)$. As $E$ is a right approximate unit we see that $\|f\|_{B} \leq\|f\| \|$. But we already know that $\|f\|\|\| f \|_{B}$ for all $f \in B$ so that $\|f\|_{B}=\|f\|$ for all $f \in B$. This and the completeness of $B$ (in \|\|$_{B}$ ) entail that $B$ is complete with respect to \|\|$\|$. Whence $B$ is closed in $\widetilde{B}^{A}$. Clearly $B$ is an ideal of $\widetilde{B}^{A}$ so the proof is complete.

Our next theorem provides information about the topology of an A-Segal algebra.

Theorem 7. Let $B$ be an A-Segal algebra. If $n>0$ then $S_{B}(\eta)=$ $S_{B}(\eta)^{A} \cap B$.

Proof. It suffices to show that $\overline{S_{B}(\eta)^{A}} \cap B \subseteq S_{B}(\eta)$. To this end let $\eta>0$ and $\epsilon>0$ be given. Choose $f \in \overline{S_{B}(\eta)^{A}} \cap \bar{B}$ and a sequence $\left\langle e_{n}\right\rangle$ from $E$ so that $\left\|f e_{n}-f\right\|_{B} \rightarrow 0$. Now choose an integer $j$ so that $\left\|f e_{j}-f\right\|_{B}$ $<\epsilon / 2$. As $f \in \overline{S_{B}(\eta)^{A}}$ we may choose a sequence $\left\langle f_{n}\right\rangle$ from $S_{B}(\eta)$ with $\left\|f_{n}-f\right\|_{A} \rightarrow 0$. Now, $\left\|f_{n} e_{j}-f e_{j}\right\|_{B} \leq\left\|f_{n}-f\right\|_{A}\left\|e_{j}\right\|_{B}$. Thus, with our choice of $j$ we may choose an integer $m$ so that $\left\|f_{m} e_{j}-f e_{j}\right\|_{B}<\epsilon / 2$. We then see that $\left\|f_{m} e_{j}-f\right\|_{B}<\epsilon$. But $f_{m} e_{j} \in S_{B}(\eta)$ since $\left\|f_{m} e_{j}\right\|_{B} \leq\left\|f_{m}\right\|_{B} \leq \eta$. Whence $f \in S_{B}(\eta)$ as $S_{B}(\eta)$ is closed in $B$.

Remark 8. We point out that Theorem 7 gives another way of showing that $B$ is closed in $\widetilde{B}^{A}$.

As it turns out there are many interesting cases when $B \neq \widetilde{B}^{A}$. Here are three examples.

Examples 9 [5]. Take $A=L^{1}(\mathbf{R})$ and consider the Segal algebras: (i) $B=L_{A}(\mathbf{R})$, the algebra of all $f \in L^{1}(\mathbf{R})$ with $f$ absolutely continuous and Df $\in L^{1}(\mathbf{R})$. The $L_{A}(\mathbf{R})$ norm is given by $\|f\|_{L_{A}}=\|f\|_{1}+\|D f\|_{1}$.

(ii) $B=T(\mathbf{R})$, the algebra of all continuous $f$ with 


$$
\|f\|_{T} \stackrel{\text { def }}{=} \sum_{n=-\infty}^{\infty} \max _{x \in[n, n+1]}|f(x)|<\infty .
$$

The $T(\mathbf{R})$ norm is \|\|$_{T}$.

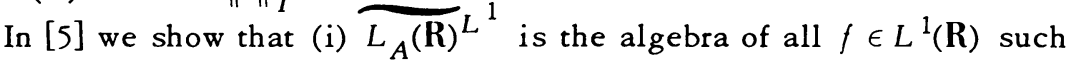
that $f$ is of bounded total variation on $\mathbf{R}$ and $\|f\|=\|f\|_{1}+V_{f}$ where $V_{f}$ is the total variation of $f$; (ii) $\widetilde{T(\mathbf{R})^{L}} L^{1}$ is the algebra of all bounded $f \epsilon$ $L^{1}(\mathbf{R})$ such that

$$
\|f\|=\sum_{k=-\infty}^{\infty} \underset{x \in[k, k+1]}{\operatorname{ess} \sup _{x \in[}|f(x)|<\infty .}
$$

In both cases we have the proper inclusion $B \not \subset \widetilde{B}^{A}$.

Now take $A=L^{1}(T)$. Define $B$ to be the set of all $f \in L^{1}(T)$ for which $\left\|f-D_{N} * f\right\|_{1} \rightarrow 0$. The $B$ norm is defined in this way:

$$
\|f\|_{B}=\operatorname{Sup}_{n \geq 1}\left\|D_{N} * f\right\|_{1} \text {. }
$$

Here $D_{N}$ denotes the Dirichlet kernel of order $N$. It is not too difficult to show that $\widetilde{B}^{L^{1}}$ consists of all $f \in L^{1}(T)$ for which $\|f \mid\|=$ $\operatorname{Sup}_{n \geq 1}\left\|D_{N} * f\right\|_{1}<\infty$. The function $f(t)=\sum_{n=2}^{\infty}(\cos n t) /(\ln n)$ shows that $B \nsubseteq \widetilde{B}^{A}$.

The preceding examples give rise to this question. What is the largest possible $A$-Segal algebra $£ A$ in which $B$ can be embedded as a closed ideal? It will be shown in Corollary 12 that $\widetilde{B}^{A}$ is the maximal such algebra.

Definition 10. We say the $A$-Segal algebra $B$ is singular $\Leftrightarrow B$ can be embedded as a closed (left, right, two-sided) ideal in some $A$-Segal algebra $B^{\prime}$ with $B \nsubseteq B^{\prime} £ A$. Segal algebras that are not singular are called nonsingular. $\widetilde{B}^{A}$

Theorem 11. If $B$ is an A-Segal algebra, then $B$ is singular $\Leftrightarrow B \nsubseteq$

Proof. If $B \unrhd \widetilde{B}^{A}$, then $B$ is singular by Theorem 6. Suppose, conversely, that $B$ is singular. Let $B^{\prime}$ be as specified in Definition 10. Let $f \in B^{\prime}$. There is a sequence $\left\langle e_{n}\right\rangle$ in $B$ such that $\left\|f e_{n}-f\right\|_{A} \rightarrow 0$. Now $\left\|f e_{n}\right\|_{B^{\prime}} \leq\|f\|_{B^{\prime}}$. But $B$ is closed in $B^{\prime}$ so there exists $M>0$ with $\|b\|_{B} \leq M\|b\|_{B}$ for all $b \in B$. Whence, taking $h=f e_{n}$, we see that $\left\|f e_{n}\right\|_{B} \leq M\left\|f e_{n}\right\|_{B^{\prime}} \leq M\|f\|_{B^{\prime}}$ for all $n$. Thus $f \in \widetilde{B}^{A}$ because $\left\|f e_{n}-f\right\|_{A} \rightarrow$ 0 (Lemma 4). We have shown that $B^{\prime} \subseteq \widetilde{B}^{A}$ and so $B \nsubseteq \widetilde{B}^{A}$ which is what we wanted to show.

Our proof of Theorem 11 shows that $\widetilde{B}^{A}$ is maximal among all subalge- 
bras of $A$ containing $B$ as a closed ideal. Indeed,

Corollary 12. If $B^{\prime}$ is an A-Segal algebra whicb contains $B$ as a closed ideal, then $B^{\prime} \subseteq \widetilde{B}^{A}$. In particular, if $\widetilde{B}^{A} \subseteq B^{\prime}$, then $B^{\prime}=\widetilde{B}^{A}$.

In contrast with 12 there may be many distinct ideals between $B$ and $\widetilde{B}^{A}$. Indeed, trivial modifications of a result due to J. Cigler [7, p. 277] obtain the following result.

Theorem 13. If $B$ is singular and $\operatorname{dimen} \operatorname{sion}\left(\widetilde{B}^{A} / B\right) \geq 2$, then there are $c$ closed ideals of $\widetilde{B}^{A}$ lying properly between $B$ and $\widetilde{B}^{A}$. Using the Hewitt, et al., module factorization theorem one can prove

Theorem 14. If $f \in \widetilde{B}^{A}$, then $f \in B \Leftrightarrow$ given any $\epsilon>0$ there exists $e(f, \epsilon)=e \in E$ so that $\|f e-f\|<\epsilon$. Furthermore, if $B^{\prime}$ is any A-Segal algebra for which $A B^{\prime}=B$, then $B^{\prime} \subseteq \widetilde{B}^{A}$.

The following corollary is an essential tool for proofs of our ideal theorems announced in [4]. We omit the easy proof.

Corollary 15. If $E$ is two sided, then $A \widetilde{B}^{A} \subseteq B$. In particular $\widetilde{B}^{A}$. $\widetilde{B}^{A} \subseteq B$ so that $\widetilde{B}^{A}$ fails to have the factorization property whenever $B$ is singular.

Our final result, is used in our analysis of the multiplier theory of commutative Segal algebras [5].

Corollary 16. Let $A=L^{1}(G)$ where $G$ is a locally compact group. Let $B=S(G)$ be a symmetric Segal algebra (commutative Segal algebras are symmetric). Then an $f \in \widetilde{S}^{L}(G)$ belongs to $S(G) \Leftrightarrow\left\|L_{y} f-f\right\|,\left\|R_{y} f-f\right\|$ both tend to zero as $y$ tends to the group identity.

Proof. Symmetry entails that $L_{y}$ and $R_{y}$ are continuous on $S(G)$ [15, p. 17]. The same proof which shows that $S(G)$ has two-sided approximate units (of $L^{1}(G)$ norm 1) [15, pp. 34-37] shows that the subalgebra of $\tilde{S}^{L^{1}}(G)$, call it $S^{\prime}(G)$, on which $L_{y}$ and $R_{y}$ act continuously has two sided approximate units. Whence by Theorem 14 we have $S^{\prime}(G)=S(G)$. On the other hand, if $f \in S(G)$ then $\left\|L_{y} f-f\right\|_{S} \rightarrow 0,\left\|R_{y} f-f\right\|_{S} \rightarrow 0$ and Theorem 6 shows that $\left\|L_{y} f-f\right\|,\left\|R x_{y} f-f\right\|_{S} \rightarrow 0$. The proof is complete.

I am grateful to Professor Richard R. Goldberg for many helpful sug gestions which have been incorporated in the final version of this paper. 


\section{REFERENCES}

1. James T. Burnham, Closed ideals in subalgebras of Banach algebras. I, Proc. Amer. Math. Soc. 32 (1972), 551-555.

2. - Closed ideals in subalgebras of Banach algebras. II: Ditkin's condition, Monatsh. Math. 78 (1974), 1-3.

3. - Segal algebras and dense ideals in Banach algebras, Proc. Internat. Conf. on Functional Analysis (Madras, India, 1973), Lecture Notes in Math., vol. 399, Springer-Verlag, Berlin and New York, 1974. (to appear).

4. - Relative completions of A-Segal algebras, Bull. Amer. Math. Soc.

5. James T. Burnham and Richard R. Goldberg, Multipliers of $L^{1}(G)$ into Segal algebras, Acta Math. Sinica (to appear).

6. - Basic properties of Segal algebras; J. Math. Anal. Appl. 42 (1973), $323-329$.

7. J. Cigler, Normed ideals in $L^{1}(G)$, Nederl. Akad. Wetensch. Proc. Ser. A 72 = Indag. Math. 31 (1969), 273-282. MR 40 \#3327.

8. B. Dunford, Segal algebras and left normed ideals, J. London Math. Soc. (to appear).

9. H. G. Feichtinger, Zur Idealtheorie von Segal-Algebren, Manuscripta Math. 10 (1973), 307-312.

10. N. Aronszajn and E. Gagliardo, Interpolation spaces and interpolation methods, Ann. Mat. Pura Appl. (4) 68 (1965), 51-117. MR 37 \# 1951.

11. Colin C. Graham, The algebraic radical of a normed ideal in $L^{1}(G)$, preprint, 1973.

12. E. Hewitt and K. A. Ross, Abstract harmonic analysis. Vol. II: Structure and analysis for compact groups. Analysis on locally compact Abelian groups, Die Grundlehren der math. Wissenschaften, Band 152, Springer-Verlag, Berlin and New York, 1970. MR 41 \# 7378; erratum, 42, p. 1825.

13. Michael Leinert, $A$ contribution to Segal algebras, Manuscripta Math. 10 (1973), 297-306.

14. M. A. Rieffel, Induced Banach representations of Banach algebras and locally compact groups, J. Functional Analysis 1 (1967), 443-491. MR 36 \#6544.

15. Hans Reiter, $L^{1}$-algebras and Segal algebras, Lecture Notes in Math., vol. 231, Springer-Verlag, Berlin and New York, 1971.

16. N. Th. Varopoulos, "The embedding of $A(E)$ into $\widetilde{A}(E)$ ", Chapter XI in: Thin sets in harmonic analysis, edited by L. A. Lindahl and F. Paulsen, Lecture Notes in Pure and Appl. Math., Dekker, New York, 1971.

DEPARTMENT OF MATHEMATICS, OKLAHOMA STATE UNIVERSITY, STILLWATER, OKLAHOMA 74074 